\title{
A prospective, randomized clinical study comparing drug eluting stent therapy and intranasal corticoid steroid therapy in the treatment of patients with chronic rhinosinusitis*
}

\author{
Rami Taulu, Argyro J. Bizaki, Jura Numminen, Markus Rautiainen \\ Department of Otorhinolaryngology, Tampere University and Tampere University Hospital, Tampere, Finland
}

Rhinology 55: 218-226, 2017

https://doi.org/10.4193/Rhino16.070

*Received for publication:

February 17, 2016

Accepted: June 26,2016

\begin{abstract}
Objectives: To conduct the first prospective, randomized controlled clinical trial comparing the efficacy of a drug-eluting stent (DES) (the Relieva Stratus ${ }^{\mathrm{TM}}$ MicroFlow Spacer) and topical intranasal corticosteroid therapy in patients with chronic rhinosinusitis (CRS).
\end{abstract}

Methods: Sixty-three adult patients with ethmoiditis were randomized into either the DES group $(n=34)$ or nasal spray group $(n=29)$. The main outcome variable was the Sinonasal Outcome Test 22, Visual Analogue Scale, nasal endoscopy, rhinometric measurements were performed at the beginning of the study, after three months and six months of follow-up.

Results: Both treatments significantly improved quality of the life with no significant difference being found between the two groups. The VAS score decreased in both groups: improvements were significant at three and six months in the nasal spray group, but in the DES group a significant difference was noted only at three months. There was a statistically significant increase in total nasal cavity volumes in the corticosteroid spray group, but not in the DES group.

Conclusion: We found that patients benefitted from DES and the corticosteroid nasal spray. We could not find any significant difference between the treatments, except the greater increase in the total nasal cavity volumes favouring the nasal spray group. Because of the very good results for the nasal spray and the much higher material and operating room costs associated with DES, we cannot recommend the use of DES over nasal spray as a monotherapeutic treatment for CRS.

Key words: chronic rhinosinusitis, ethmoiditis, drug eluting stent, Relieva Stratus Micro Flow Spacer, nasal corticosteroids

\section{Introduction}

Chronic rhinosinusitis (CRS) is a common health problem that affects $5-15 \%$ of the Western population ${ }^{(1-3)}$. CRS has a major impact on quality of life, comparable to chronic diseases such as ischemic heart disease, congestive heart failure, and back pain ${ }^{(4)}$. It causes a substantial economic burden to society and health care systems ${ }^{(5)}$. CRS is a wide general term describing a group of disorders characterized by chronic inflammation of the mucosa of the nose and paranasal sinuses that share the same symptomology. The goals of the treatment include reduction of mucosal oedema, re-establishment of sinus ventilation, and eradication of infecting pathogens. Multiple therapies are available for the management of CRS, including topical and systemic glucocorticoids, long- and short-term antibiotics, nasal saline irrigations or sprays, antileukotriens, anti-interleukin therapy, and endoscopic sinus surgery (ESS).

Intranasal glucocorticoid therapy is the first-line treatment for CRS. There is good evidence for the efficacy of topically adminis- 
tered glucocorticoid therapy when treating CRS with or without nasal polyps ${ }^{(6-8)}$. The topical administration route is safe and poses only minor side effects, such as mucosal irritation, crusting, and minor nose bleeds ${ }^{(7)}$. The complexity of the sinus anatomy and the mucosal oedema of CRS patients are major problems in drug delivery to the affected paranasal sinus mucosa ${ }^{(9)}$. Several large prospective studies have shown that ESS is an effective and safe method of treatment for patients with CRS when drug therapy has failed ${ }^{(10,11)}$. The goal of ESS is to ensure the ventilation of the diseased paranasal sinuses and the restoration of the mucociliary function. One of the advantages of ESS is that delivery of the topical glucocorticoids to the paranasal sinuses is easier postoperatively ${ }^{(12,13)}$.

Recently published clinical trials have demonstrated that the application of a bioabsorbable mometasone eluting stent (the Propel $^{\circledast}$ Intersect ENT) in patients that have been undergone ESS is safe and improves surgical results by minimizing the occurrence of inflammation, adhesions, and polypoid tissue formation ${ }^{(14-16)}$. The Relieva Stratus ${ }^{\mathrm{TM}}$ Micro-flow Spacer (Relieva Stratus; Acclarent Inc., CA, USA) is a method for delivering glucocorticoids directly to affected paranasal sinuses. It has been used to treat frontal, ethmoidal, and sphenoidal sinuses in a preoperative setting. It is an interesting idea to use this drug eluting stent (DES), which is introduced with a trocar-based delivery system, as a monotherapeutic anti-inflammatory treatment to avoid surgery and preserve normal ethmoid sinus anatomy. Cadaveric studies have demonstrated that insertion of the Relieva Stratus into the ethmoidal sinus using fluoroscopy is relatively safe and easy ${ }^{(17)}$. An image guided surgery system (IGS) should be used for the guided insertion instead of fluoroscopic insertion, because the former is a faster, safer, and more exact procedure, and it does not use ionizing radiation ${ }^{(18)}$.

In an initial report, Catalano et al. ${ }^{(19)}$ evaluated the short-term outcomes and safety of the Relieva Stratus infused with triamcinolone acetonide. They followed 23 patients for six months and reported that the Relieva Stratus was a safe and effective method of treatment for chronic ethmoid sinus disease. Outcomes were evaluated by observing changes in the 20-item Sino-Nasal Outcome Test (SNOT-20) and Lund-Mackay (LM) scores.

This study is a prospective, randomized controlled clinical trial on patients diagnosed with CRS with ethmoidal involvement verified by CBCT scan findings. Our objectives were to compare the efficacy of the DES to the standard non-invasive treatment with corticosteroid nasal spray and to study the safety and potential side effects of the Relieva Stratus.

\section{Materials and methods}

Subjects

A total of 63 patients were prospectively recruited to a randomized controlled clinical trial at the Department of Otorhinolaryngology at Tampere University Hospital, Finland over a 42-month period starting from December 2010 and ending in June 2014. All the recruited patients were referred from outpatient clinics. Patients did not receive any financial compensation for their participation in this study. Informed consent was obtained from all patients in advance. The trial protocol was approved by the Ethics Committee of Pirkanmaa Hospital District, Tampere University Hospital.

Inclusion criteria were: 1) CRS diagnosed as outlined by the European position paper on rhinosinusitis and nasal polyps 2007 $(20), 2$ ) adequate medical treatment of the CRS for at least three months without a satisfactory result, 3 ) age over 18 years and less than 65 years, 4) CRS of ethmoid sinuses, confirmed with a CBCT scan and an LM score of at least 2 for ethmoid sinuses, and 5) fulfilment of the indications for sinus surgery according to Finnish guidelines and indications for surgical treatment ${ }^{(21)}$.

Exclusion criteria were as follows: 1) previous sinus operations, 2) ASA hypersensitivity, 3) diabetes or any other severe systemic disease, 4) glaucoma, 5) pregnancy upon enrolment to the study, 6) an LM score of more than 1 in the maxillary, frontal or sphenoidal sinus, 7) distance from the face of the ethmoid bulla to the face of the sphenoid sinus less than $20 \mathrm{~mm}$, and 8) nasal polyposis if the polyps were growing beyond the medial meatus according to an endoscopic view.

The study protocol included four to five study visits. The first visit was an enrolment and randomization visit, during which the informed consent and history were obtained, direct nasal endoscopy was performed, and the CBCT scans were evaluated. From this point began a four week wash-out period. The patients were forbidden from using any medication containing corticosteroids. Any other medication to treat the symptoms of the CRS was allowed (no-one used long term, low-dose macrolide antibiotic drugs). At the next visit, both groups were evaluated using SNOT22, VAS, RMM, AR, and direct nasal endoscopy. In the DES group, all of the patients had bilateral stent placement under general anaesthesia. Patients in the nasal spray group were instructed as to the ideal way to administer the nasal spray. Any other drugs containing corticosteroids were forbidden in both groups for the duration of the trial. The DES was removed after four weeks at an outpatient clinic under local anaesthesia. Follow up was scheduled at three and six months after treatment; the patients were again evaluated using SNOT22, VAS, $\mathrm{RMM}, \mathrm{AR}$, and direct nasal endoscopy. Usage of antibiotics was documented and an additional sinus CBCT scan was taken at six months after treatment. Patients were regularly followed up for any adverse effects.

\section{Study groups}

Patients were randomized into the DES or the intranasal corticosteroid spray group using the MINIM MS DOS program (a program for randomization in clinical trials) ${ }^{(22)}$. It does allocation by minimisation and runs interactively through the study. 
Groups were randomized with the following parameters: age, sex, asthma, nasal polyposis, and use of tobacco. The sample size was calculated based on paired t-test. The value for average SNOT22 change was set to 12.6, 20.0 was used as standard deviation ${ }^{(23)}$. With alpha of 0.05 and power of 0.8 the calculation suggests that approximately 22 study patients are needed to see the SNOT22 change as statistically significant. A drop-out of patients in follow-up had to be taken into account, so approximately 30 patients in both groups (a total of 60 ) were required.

\section{Sino Nasal Outcome Test-22 Quality of Life Questionnaire} The SNOT22 is a validated, rhinosinusitis-specific quality of life instrument that contains 22 individual questions about nasal symptoms and quality of life ${ }^{(23)}$. Patients completed the questionnaire after the wash out period, at three months, and at six months. According to a previous validation study, we considered the minimally important difference - the smallest change in the SNOT22 score that can be detected in a patient - to be 8.9 points ${ }^{(23)}$.

\section{Visual Analogue Scale}

Patients were asked to score using the visual analogue scale (0$10 \mathrm{~cm}$ ) the question "How troublesome are your CRS symptoms?" $(0 \mathrm{~cm}=$ not troublesome at all to $10 \mathrm{~cm}=$ worst thinkable $)$. VAS scoring was performed at every visit.

\section{Acoustic rhinometry and rhinomanometry}

AR evaluates nasal obstruction by analysing reflected sound waves introduced through the nostrils. It produces an image that reflects variations in the cross-sectional dimensions of the nasal cavity and closely approximates nasal cavity volume and minimal cross-sectional area. It is easy to perform, non invasive, and does not require significant patient co-operation. It can be used to demonstrate the reduction of the inflammation as a result of medical and surgical intervention ${ }^{(24)}$. We analysed the change in the total volume (between 2 to $5 \mathrm{~cm}$ from the nostril) of the nasal cavity ${ }^{(25)}$.

RMM, a measurement of nasal airway resistance, was performed at every study visit. It is a tool to determine the degree of airflow obstruction before and after surgical procedures and pharmacological interventions. In CRS, this method can be used to confirm if the improvement in nasal congestion is the result of a reduction in inflammation ${ }^{(26)}$. In this study, we examined the change in the total inspiratory nasal resistance as a sign of the extent of mucosal inflammation.

\section{Direct nasal endoscopy}

In the study, we used the EPOS guideline for endoscopy scoring $(0=$ absence of polyps, $1=$ polyps in middle meatus only, $2=$ polyps beyond middle meatus but not blocking the nose completely, $3=$ polyps completely obstructing the nose), oedema ( 0

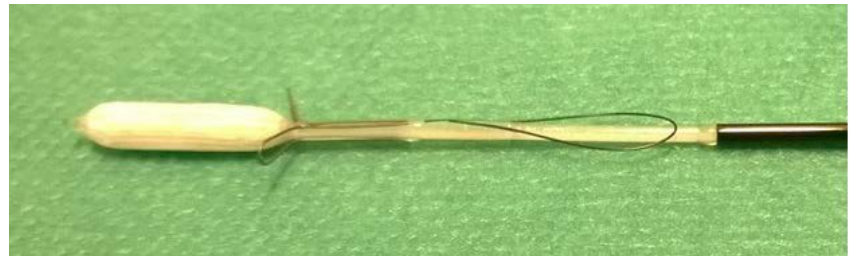

Figure 1. The Relieva Stratus ${ }^{\mathrm{TM}}$ MicroFlowSpacer filled with triamcinoloneacetonide.

$=$ absent, $1=$ mild, $2=$ severe $)$, discharge $(0=$ no discharge, $1=$ clear, thin discharge, 2 = thick, purulent discharge), scarring $(0$ =absent, $1=$ mild, $2=$ severe $)$, and crusting $(0=$ absent, $1=$ mild, 2 = severe) ${ }^{(20)}$. The patient's nasal cavities were examined with an endoscope and graded by the same operator at every visit.

\section{Cone beam computed tomography of paranasal sinuses} In recent years, $\mathrm{CBCT}$ scanning has become a competitor to traditional multi-row computed tomography. The major advantage is the lower radiation exposure, which can be as low as one half to one quarter of a standard multi-row CT scanner, and cost is also much lower.

An LM score was used to evaluate the CBCT scans. Each group of sinuses (maxillary, anterior ethmoids, posterior ethmoids, frontal, and sphenoidal) were analysed in cross-sectional images and scored as either completely clear (0), partly opaque (1), or completely opaque (2), and the opacity of the ostiomeatal complex was scored ( 0 or 2 ). The scores were added together, resulting in a maximum complete score of 12 per side. The LM score was analysed by the same doctor.

\section{The drug eluting stent}

The Relieva Stratus is a temporarily implanted drug-eluting stent. The Relieva Stratus device was introduced in 2009 as a minimally invasive surgical tool to treat chronic ethmoidal sinusitis ${ }^{(27)}$. The stent is inserted into the ethmoidal sinus complex using an endoscopic view with the aid of fluoroscopy or IGS. The reservoir section of the device contains hundreds of micropores that slowly release a therapeutic agent into the ethmoidal complex (Figure 1). This local and targeted method of drug delivery ensures a high concentration of the anti-inflammatory agent directly into the diseased mucosa. In the US, the FDA has currently approved the Relieva Stratus loaded only with sterile saline. In Europe, the device has a CE Mark approval that also covers the use of triamcinolone acetonide $(40 \mathrm{mg} / \mathrm{ml})$. If both sides are implanted, a total amount of $24 \mathrm{mg}$ triamcinolone will leak from the pores over a period of four weeks. Triamcinolone acetonide is widely used and thus its potential side effects are well known ${ }^{(28)}$.

The manufacturer of the device recommends that $0.31 \mathrm{ml}$ of sterile saline or triamcinolone acetonide injectable solution (40 


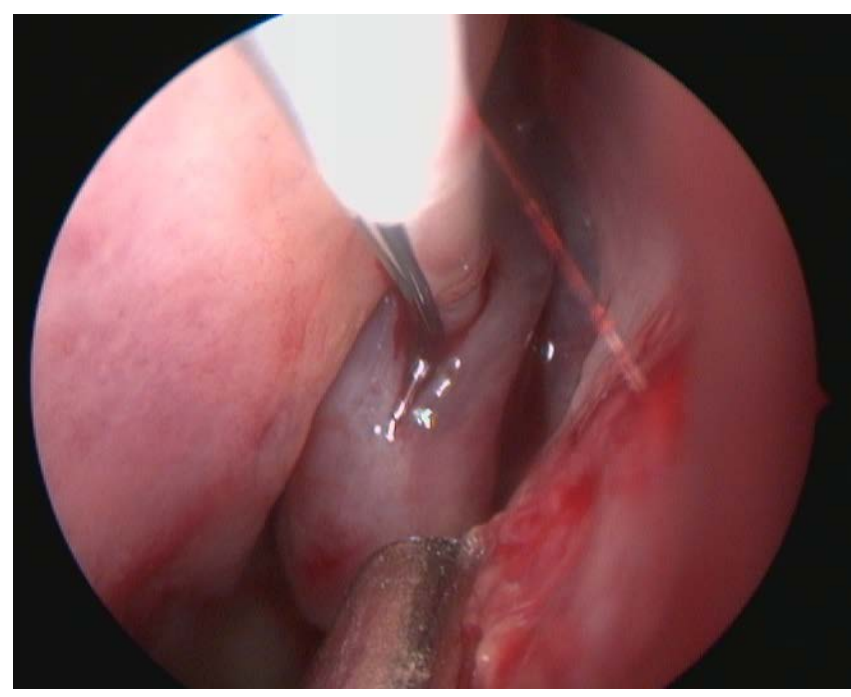

Figure 2. Endoscopic view of the right bulla ethmoidalis. With the help of the IGS insertion, the insertion point can safely be more cranial.

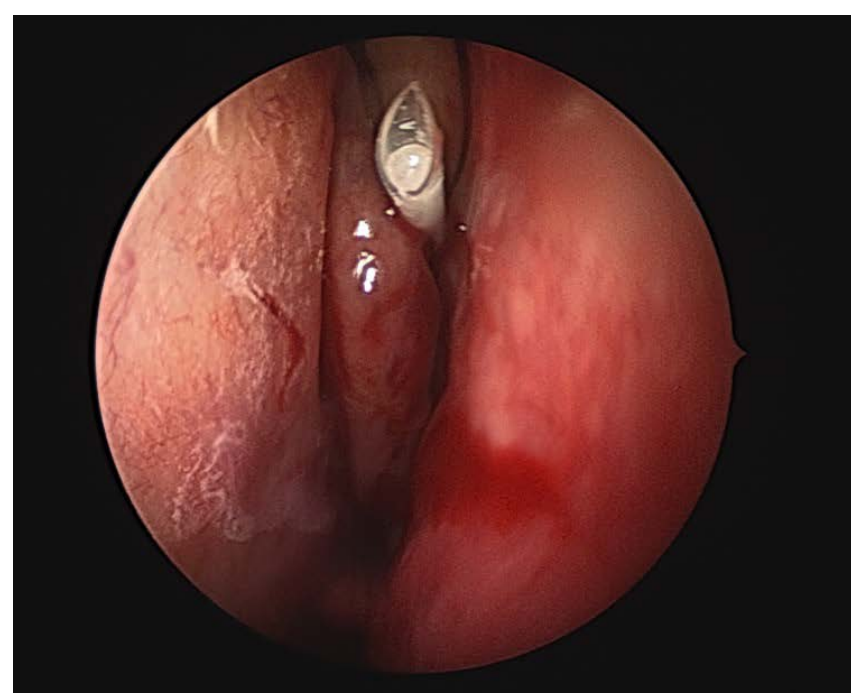

Figure 3. Endoscopic view of the final position of the Relieva Stratus ${ }^{\mathrm{TM}}$, MicroFlow Spacer. Only the cut end of the catheter shaft is visible in the picture (left side). $\mathrm{mg} / \mathrm{ml}$ ) is injected into the catheter ${ }^{(29)}$. The shaft of the catheter is then cut and the MicroFlow Spacer is left in the ethmoid sinus (Figures 2 and 3). After four weeks, the stent is extracted at an outpatient clinic under local anaesthesia by simply removing it with Blakesley forceps or a similar instrument.

In our hospital the cost of the MicroFlow Spacer is $€ 399$ and the cost of the deployment guide is $€ 175$. When treating both ethmoidal sinuses the total cost of the devices is $€ 975$.

$C$-arm fluoroscopy guidance was employed for eleven patients and optical IGS-assisted (BrainLAB Kolibri image-guided surgery system) insertions were performed on the other 17 patients. All the insertions of the DES were performed by the same surgeon.

\section{Intranasal corticosteroid spray}

Triamcinolone acetonide is extensively used in different kind of drugs and its potential side effects are very well known ${ }^{(28)}$. The control group using the triamcinolone acetonide nasal spray (Nasacort ${ }^{\circledast} 55 \mathrm{ug} /$ dose) applied two doses/day for six months. The use of nasal corticosteroid sprays predisposes to minor side effects, with epistaxis being the most common. Patients were instructed how to apply the nasal spray inside the nose and they were also informed that the weight of the spray bottle would be controlled at the end of the study. The drugs (Kenalog $40^{\circledR}$ and Nasacort ${ }^{\oplus}$ ) used in this trial were bought from Tampere University Hospital's pharmacy.

\section{Statistical analyses}

Statistical analysis and graphical representation of the results were performed using the SPSS 21 software (SPSS Inc., Chicago, IL, USA). The primary variable was the SNOT22 score. Improvement in quality of life was analysed with Wilcoxon Signed Rank test. Differences between the groups were analysed with the Mann-Whitney $U$ test. $P$ values smaller than 0.05 were considered significant.

\section{Results}

Subjects

A total 63 patients were enrolled in the study and were randomized into the DES group $(n=34)$ and nasal spray group $(n=$ 29). The first two DES patients were excluded, because there was too little of the corticosteroid solution inside the reservoir of the stent. Four patients in the DES group were lost to the follow-up (one patient had severe CRS symptoms and was operated on immediately after the removal of the stent, one patient was obliged to use another corticosteroid drug because of another unrelated disease, one patient dropped out having been diagnosed with an unrelated serious disease, and one patient dropped out for personal reasons). Finally, 57 patients ( 18 males and 39 females) were included in the analysis, 28 to the DES group and 29 to the control group treated with the nasal corticosteroid spray.

No significant differences were identified between the groups when comparing the baseline demographic characteristics (Table 1). The main symptoms were nasal blockage/obstruction/ congestion (96.5\%), nasal discharge (anterior/posterior drip; $86.0 \%)$, facial pain/pressure (80.7\%), and reduction or loss of the sense of smell (50.9\%) (Table 2). The use of saline nasal irrigation varied greatly; some of the patients used it daily and others only when they had many symptoms.

No statistically significant difference was found between the groups at baseline in the SNOT22 scores, VAS scores, endoscopic scores, LM scores, total nasal volumes, or the measurements of 
Table 1. Patient demographic characteristics.

\begin{tabular}{|c|c|c|c|}
\hline & DES $(n=28)$ & Nasal spray $(n=29)$ & Both groups $(n=57)$ \\
\hline Mean age $\pm S D$ (years) & $42.9 \pm 11.6$ & $41.1 \pm 12.6$ & $42.0 \pm 12.1$ \\
\hline $\operatorname{Sex}(M / F)$ & $8 / 20$ & $10 / 19$ & $18 / 39(31.6 \% / 68.4 \%)$ \\
\hline Smokers & 9 & 10 & $19(33.3 \%)$ \\
\hline Mean duration of symptoms (months) & $59.4 \pm 63.5$ & $55.3 \pm 63.3$ & $58.3 \pm 62.3$ \\
\hline Polyps & 5 & 4 & $9(15.8 \%)$ \\
\hline Allergy & 8 & 13 & $21(36.8 \%)$ \\
\hline Asthma & 4 & 5 & $9(15.8 \%)$ \\
\hline Usage of saline nasal irrigation & 19 & 14 & $33(57.9 \%)$ \\
\hline Mean duration of use of steroid spray (months) before the study & $20.9 \pm 35.8$ & $14.1 \pm 26.0$ & $17.4 \pm 31.1$ \\
\hline Number of courses of antibiotics/patient (six months before the study) & $2.1 \pm 2.1$ & $2.2 \pm 2.1$ & $2.2 \pm 2.1$ \\
\hline
\end{tabular}

Table 2. Main symptoms.

\begin{tabular}{|c|c|c|c|}
\hline & DES ( $n=28)$ & Nasal spray $(n=29)$ & Both groups $(n=57)$ \\
\hline Nasal blockage/ obstruction/congestion & $27(96.4 \%)$ & $28(96.6 \%)$ & $55(96.5 \%)$ \\
\hline Nasal discharge & $24(85.7 \%)$ & $25(86.2 \%)$ & $49(86.0 \%)$ \\
\hline Facial pain/pressure & $23(82.1 \%)$ & $23(79.3 \%)$ & $46(80.7 \%)$ \\
\hline Reduction or loss of smell & $11(39.3 \%)$ & $18(62.1 \%)$ & $29(50.9 \%)$ \\
\hline
\end{tabular}

Table 3. Baseline data.

\begin{tabular}{|c|c|c|c|c|c|c|}
\hline & \multicolumn{2}{|c|}{ DES } & \multicolumn{2}{|c|}{ Nasal spray } & \multicolumn{2}{|c|}{ Both groups } \\
\hline & Mean & SD & Mean & SD & Mean & SD \\
\hline SNOT22 & 43.9 & \pm 16.9 & 42.8 & \pm 14.2 & 43.3 & \pm 15.5 \\
\hline VAS & 5.3 & \pm 2.3 & 5.7 & \pm 2.6 & 5.5 & \pm 2.5 \\
\hline AR, total volume $\left(\mathrm{cm}^{3}\right)$ & 7.7 & \pm 2.3 & 6.8 & \pm 2.0 & 7.3 & \pm 2.2 \\
\hline $\begin{array}{l}\text { RMM, total inspiratory nasal } \\
\text { resistance }\left(\mathrm{Pa} / \mathrm{cm}^{3} / \mathrm{s}\right)\end{array}$ & 0.23 & \pm 0.25 & 0.36 & \pm 0.35 & 0.30 & \pm 0.31 \\
\hline Endoscopic score & 2.0 & \pm 1.7 & 1.9 & \pm 2.0 & 2.0 & \pm 1.8 \\
\hline LM score (before the trial) & 10.6 & \pm 3.2 & 11.1 & \pm 2.6 & 10.9 & \pm 2.9 \\
\hline
\end{tabular}

the total mean airway resistance (Table 3).

\section{SNOT22}

At the three- and six-month follow-up visits, the total SNOT22 score showed a significant improvement in both groups, with no significant difference between the two groups. In the DES group, the mean change from the baseline score was $-17.0 \pm 16.7$ $(p<0.001)$ after three months and $-12.0 \pm 13.9(p<0.001)$ after six months. The same values in the nasal spray group were -10.1 $\pm 13.8(p=0.001)$ and $-10.2 \pm 15.9(p=0.002)$, respectively. There was a tendency for patients in the DES group to benefit more from the treatment after three months compared to the nasal spray group, but the difference was not statistically significant $(p=0.078)$ (Figure 4).

\section{VAS}

The average VAS score decreased in both groups. The mean changes were significant at three months $(1.4 \pm 2.5, p=0.006)$ and at six months $(-1.4 \pm 3.1, \mathrm{p}=0.026)$ in nasal spray group, but in the DES group a significant difference was noted only at three months $(-1.9 \pm 2.4, p=0.001)$. There were no significant differences between the groups.

\section{Acoustic rhinometry}

The total nasal volumes in the nasal spray group increased significantly at three months $\left(1.2 \mathrm{~cm}^{3} \pm 2.3 \mathrm{~cm}^{3}, \mathrm{p}=0.001\right)$ and six months $\left(1.4 \mathrm{~cm}^{3} \pm 2.0 \mathrm{~cm}^{3}, \mathrm{p}=0.002\right)$. Total nasal volumes in the stent group did not show any significant changes from the baseline measurements. The differences between the groups were 


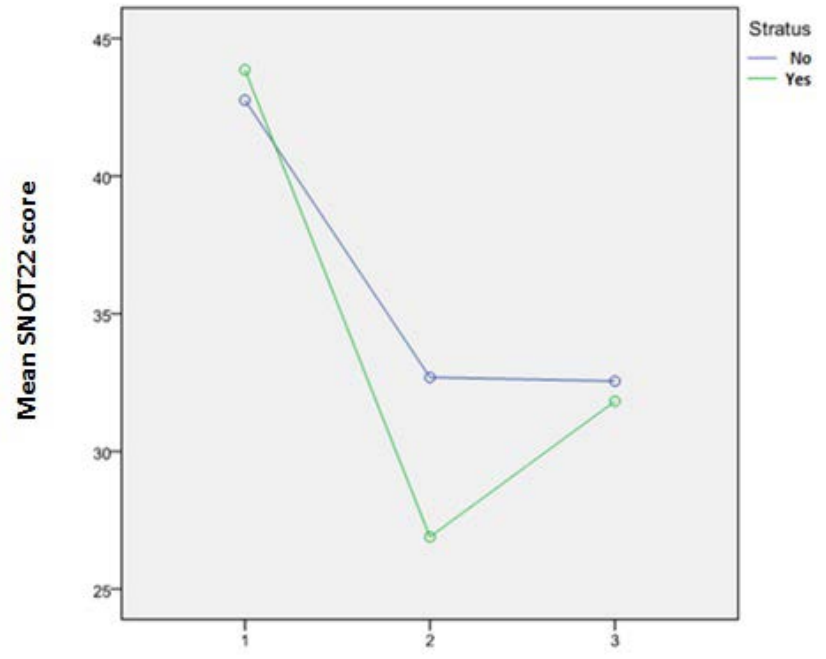

Study visit number

Figure 4. After three months, the SNOT22 scores were lower in the stent group compared to the nasal spray group. However, the difference was not statistically significant $(p=0.078)$.

significant at three months $(p=0.025)$ and six months $(p=0.016)$.

\section{Rhinomanometry and the endoscopic score}

There were no (significant) changes in measurements by RMM or the endoscopic score either at three months or six months. No statistically significant difference was found between the groups either.

\section{LM score}

In the DES group, the change in LM score was almost statistically significant $(-1.9 \pm 4.4, \mathrm{p}=0.056)$ when comparing the values before the study to the values at the end of the study. There were no significant differences between the groups.

\section{Use of antibiotics}

The use of the antibiotics was significantly reduced in both groups (in the DES group $-1.4 \pm 2.6, p=0.013$ and in the nasal spray group $-1.8 \pm 2.1, \mathrm{p}<0.001$ ) when comparing the mean number of courses of antibiotics to the six-month period before the beginning of the study and the trial period. No difference was found between the groups.

\section{Adverse events}

There were no significant immediate or delayed complications in either group. No DES needed to be removed before the end of the four-week treatment period. After the insertion of the stent, some patients experienced minor sensations of local irritation. When the implant was removed, there was minor crusting $(n=2)$ and a small amount of purulent discharge $(n=3)$ around the cut end of the shaft of the catheter in the endoscopy in some patients. One patient needed antibiotics to treat these symptoms. Four patients had minor nose bleeds, which lasted one to two days, after the insertion of the implant; however no interventions were needed to treat them. Two patients from the nasal spray group complained of dryness of the nose.

\section{Discussion}

Current standard surgical treatment for chronic inflammatory disease of the ethmoid sinus is endoscopic ethmoidectomy performed with a microdebrider, through-cutting forceps, or a combination of the two. However, the widespread use of these tools has been known to cause adverse effects such as postoperative inflammation, middle meatal scarring, and very rarely complications as severe as cerebrospinal fluid leak, orbital emphysema, and potential visual change or loss of vision. A topical steroid is a beneficial treatment for CRS with and without polyps, but the main disadvantage with the use of the nasal sprays and drops is suboptimal drug delivery to the paranasal sinuses. Other problems with use of nasal sprays include the wrong dosing technique and a lack of motivation to use the medication as regularly and long-lastingly as recommended. The Relieva Stratus is an interesting mini-invasive option to deliver strong corticosteroid medication directly to the diseased paranasal sinus mucosa. Cadaveric studies have demonstrated that insertion of the Relieva Stratus into the ethmoidal sinus using fluoroscopy is relatively safe and easy ${ }^{(17)}$. There is only one published complication involving the Relieva Stratus in the literature; the DES had been wrongly placed through the lamina papyracea into the orbit. Despite the removal of the device, the pupil of patient's affected eye remained dilated ${ }^{(30)}$. The U.S. Food and Drug Administration (FDA) has reported one skull base injury caused by the insertion of the Relieva Stratus deployment guide through the posterior ethmoidal roof. The cerebrospinal fluid leak was detected and treated without further complications ${ }^{(31)}$. There are no prospective, randomized controlled trials addressing the efficacy of the Relieva Stratus or other corticosteroid eluting stents in patients being considered for endoscopic surgery. In our prospective, randomized clinical study, we compared the efficacy of the Relieva Stratus to ideally dosed, regular, long-term use of nasal corticosteroid spray in patients with CRS.

We found that the quality of life measured by SNOT22 score improved significantly in both treatment groups at three and six months of observation. In addition, the VAS score decreased in both groups. The observed benefits of the nasal corticosteroid spray treatment measured by VAS were evident at three months and six months, but in the DES group a significant improvement was noted only at the three month time point. There was a statistically significant increase in the total nasal cavity volumes measured with AR in the corticosteroid spray group at three months and six months, but not in the stent group. The most surprising result was the significant increase of the quality of life 
Table 4. Three-month change in measurements of the DES and nasal spray group.

\begin{tabular}{|c|c|c|c|c|c|}
\hline \multirow[b]{2}{*}{ Parameter group } & \multicolumn{2}{|c|}{ DES change from baseline } & \multicolumn{2}{|c|}{ Nasal spray change from baseline } & \multirow{2}{*}{$\begin{array}{c}\text { Statistical } \\
\text { difference be- } \\
\text { tween the groups }\end{array}$} \\
\hline & Mean & SD & Mean & SD & \\
\hline SNOT22 & $-17.0\left(p<0.001^{*}\right)$ & \pm 16.7 & $-10.1\left(p=0.001^{*}\right)$ & \pm 13.8 & $p=0.078^{* *}$ \\
\hline VAS & $-1.9\left(p=0.001^{*}\right)$ & \pm 2.4 & $-1.4\left(p=0.006^{*}\right)$ & \pm 2.5 & NS \\
\hline AR, total nasal volume $\left(\mathrm{cm}^{3}\right)$ & -0.33 (NS) & \pm 2.8 & $1.2\left(p=0.001^{*}\right)$ & \pm 2.3 & $\mathrm{p}=0.025^{* *}$ \\
\hline $\begin{array}{l}\text { RMM, total inspiratory nasal resistance } \\
\left(\mathrm{Pa} / \mathrm{cm}^{3} / \mathrm{s}\right)\end{array}$ & 0.03 (NS) & \pm 0.29 & -0.05 (NS) & \pm 0.34 & NS \\
\hline Endoscopic score & -0.1 (NS) & \pm 1.9 & 0.4 (NS) & \pm 1.8 & NS \\
\hline
\end{tabular}

*Wilcoxon signed ranks test, **Mann-Whitney $U$ test.

Table 5. Six-month change in measurements of the DES and nasal spray group.

\begin{tabular}{|c|c|c|c|c|c|}
\hline \multirow[b]{2}{*}{ Parameter group } & \multicolumn{2}{|c|}{ DES change from baseline } & \multicolumn{2}{|c|}{ Nasal spray change from baseline } & \multirow{2}{*}{$\begin{array}{c}\text { Statistical } \\
\text { difference be- } \\
\text { tween the groups }\end{array}$} \\
\hline & Mean & SD & Mean & SD & \\
\hline SNOT22 & $-12.0\left(p<0.001^{*}\right)$ & \pm 13.9 & $-10.2\left(p=0.002^{*}\right)$ & \pm 15.9 & NS \\
\hline VAS & -0.7 (NS) & \pm 2.8 & $-1.4\left(p=0.026^{*}\right)$ & \pm 3.1 & NS \\
\hline AR total nasal volume $\left(\mathrm{cm}^{3}\right)$ & $-0.11(N S)$ & \pm 2.6 & $1.4\left(p=0.002^{*}\right)$ & \pm 2.0 & $\mathrm{p}=0.016^{* *}$ \\
\hline $\begin{array}{l}\text { RMM, total inspiratory nasal resistance } \\
\left(\mathrm{Pa} / \mathrm{cm}^{3} / \mathrm{s}\right)\end{array}$ & 0.12 (NS) & \pm 0.58 & -0.08 (NS) & \pm 0.25 & NS \\
\hline Endoscopic score & 0.1 (NS) & \pm 1.5 & -0.2 (NS) & \pm 2.2 & NS \\
\hline LM score & $-1.9\left(p=0.056^{*}\right)$ & \pm 4.4 & -0.7 (NS) & 2.7 & NS \\
\hline $\begin{array}{l}\text { Course of antibiotics/patient six } \\
\text { months before trial vs follow-up time }\end{array}$ & $-1.4\left(p=0.013^{*}\right)$ & \pm 2.6 & $-1.8\left(p<0.001^{*}\right)$ & \pm 2.1 & NS \\
\hline
\end{tabular}

*Wilcoxon signed ranks test, **Mann-Whitney $U$ test.

in the nasal spray group, although these patients had already used corticosteroid nasal spray before the study without notable relief of symptoms. The use of the nasal spray increased the total volume of the nasal cavities, most likely by decreasing the mucosal swelling. These results emphasize the importance of the ideal dosing technique, motivation, and regular, long-term administration of the topical corticosteroid medication.

Our hypothesis was that the patients in the DES group would experience better relief of symptoms compared to patients in the nasal corticosteroid group. We found no support for this hypothesis. Instead, when we analysed nasal volumes in AR, we found that patients in the nasal spray group had significantly better results compared to patients in the stent group. The SNOT22 scores between the groups were similar at the beginning and at the end of the study. However, we found that at three months, the SNOT22 scores were lower in the stent group compared to the nasal spray group. The difference was not statistically significant $(p=0.078)$. The fat-soluble triamcinolone acetonide continues to have an effect on the mucosa after the Relieva Stratus has been removed from ethmoid sinuses. It is likely that when reaching the six-month time point, the effect of the corticosteroid of the Relieva Stratus has already diminished and the difference between the groups disappears. This notion is also supported by the finding that there was no statistically significant improvement in VAS score at six months in the stent group. Like asthma, CRS is a chronic disease and corticosteroid medication simply reduces the inflammation caused by various aetiologies and relieves the symptoms; it does not provide a definitive cure for the disease.

There was no placebo group in our study. We wanted to compare the efficacy of the Relieva Stratus to a proven conservative treatment. The national comparative audit of surgery for nasal polyposis and chronic rhinosinusitis was a prospective cohort study of 3,128 adult patients that underwent sinonasal surgery in England and Wales ${ }^{(23)}$. Hopkins et al. evaluated 2,284 preoperative and postoperative SNOT22 scores for psychometric validation. SNOT22 was able to discriminate between patients known to suffer from CRS and a group of healthy controls. They found that the minimum difference detectable with the SNOT22 scoring scheme was 8.9 points ${ }^{(23)}$. In that study, the mean preoperative SNOT22 score of patients undergoing primary surgery was 39.6, which is similar to our results (43.3). Furthermore, in the present study, the mean change in SNOT22 scores after the treatment period was $>10$ in both groups. 
In our study, the baseline LM scores were quite low $(10.6 \pm 3.2$ in the DES group and $11.1 \pm 2.6$ in the nasal spray group), because we excluded from the study those patients that had an LM score of more than 1 in the maxillary, frontal, or sphenoidal sinuses. Because the target area of the Relieva Stratus is the ethmoidal cells, we found it unethical to treat patients with severe sinus pathology in maxillary, frontal, and sphenoidal sinuses (LM score 2) only with the DES. Therefore, in our study the maximum LM score was 18 instead of the normal maximum of 24. In the operating instructions ${ }^{(29)}$ provided by Acclarent Inc. and also in previous studies, it was recommended that $0.31 \mathrm{ml}$ of the therapeutic agent should be injected into the catheter. We found this amount to be too small because of the dead space of the catheter shaft. To ensure that the Spacer reservoir is filled one must inject at least $0.6 \mathrm{ml}$ of the solution into the catheter.

Any excess solution leeches out into the ethmoidal cavities. This technical detail was the reason why we excluded the first two patients in the Relieva Stratus group.

The six month follow-up time might be too short to evaluate the long-term effect of the DES. All the patients who participated in the study had severe symptoms and were candidates for surgery, so a six month follow-up period was considered feasible and we considered a longer follow-up to be unethical.

When comparing antibiotic use six months before the study and then during the study, we found a significant decrease in both groups. It is most likely that before the study CRS symptoms were treated as acute bacterial rhinosinusitis. At the beginning of the study, the patients were educated about the inflammatory nature of CRS and the poor efficacy of short term antibiotic treatment. After this, the use of the antibiotics decreased dramatically.

In our study, the mean SNOT22 score change after three months in the patients treated with DES was 17.0, which is quite similar to the results of the national comparative audit of surgery for nasal polyposis and chronic rhinosinusitis study ${ }^{(23)}$, where the mean SNOT22 score was 16.2 points lower after the ESS surgery than preoperatively. Of course, the results of these two studies cannot be compared completely because of the methodological differences between the study designs, but our results give an indication that Relieva Stratus is an effective therapy in treating patients with CRS.

The postoperative treatment of CRS after ESS includes local corticosteroid therapy ${ }^{(20)}$. In our study, the DES has been investigated as a monotherapeutic treatment. It is possible that combined therapy with the DES and the nasal corticosteroid spray therapy could be even more efficient.

Targeted administration of corticosteroids using the DES might be a considerable tool for the treatment of chronic ethmoiditis instead of ESS in cases where we want to avoid systemic corticosteroids or surgery, or where the diseased area in the ethmoidal sinuses is highly localized.

\section{Conclusion}

Patients benefitted from the Relieva Status as well as from the corticosteroid nasal spray, and quality of life improved for both groups. With the exception of a greater improvement in nasal volumes in the nasal spray group, no significant difference was observed between the treatment methods. The insertion of the Relieva Stratus requires an operation room involving fluoroscopy or the IGS system, and the material costs of the device is considerably higher compared to the use of the corticosteroid spray. Overall, compared to the corticosteroid nasal spray therapy, there was no remarkable advantage in using the Relieva Stratus stent technique as a monotherapy that would justify its increased costs and potential side effects.

The surprising result was the significant increase of the quality of the life also in the nasal spray group, since patients had already used corticosteroid nasal spray before the study without any significant results. This result emphasizes the importance of the ideal dosing technique, motivation, and the regular, longterm administration of the topical corticosteroid therapy.

\section{Acknowledgements}

To research nurse Piitu Oksanen for ensuring patients'smooth coordination and support throughout the study and to Niko Sillanpää MD, PhD for scientific and linguistic help.

\section{Authorship contribution}

RT: Principal researcher of the study, study design, recruitment of new patients, clinical examination of patients before and after the intervention, insertions of the drug eluting stents, collection and analysis of data, writing of the manuscript. AB: Manuscript review. JN: Study design, manuscript review. MR: Study design, manuscript review.

\section{Conflict of interest}

None of the authors had any conflict of interest with any finan $\neg$ cial organization. This study was not sponsored by Acclarent Inc. or any other pharmaceutical companies or any other personal or institutional funds.

\section{References}

1. Blackwell DL, Collins JG, Coles R. Summary health statistics for U.S. adults: Nationa Health Interview Survey, 1997. Vital Health Stat 10 2002; 205: 1-109.
2. Hastan D, Fokkens WJ, Bachert C, et al. Chronic rhinosinusitis in Europe-an underestimated disease. A GA(2)LEN study. Allergy 2011; 66(9): 1216-23.

3. Pilan RR, Pinna FR, Bezerra TF, et al.
Prevalence of chronic rhinosinusitis in Sao Paulo. Rhinology 2012; 50(2): 129-38.

4. Gliklich RE, Metson R. The health impact of chronic sinusitis in patients seeking otolaryngologic care. Otolaryngol Head Neck 
Surg. 1995; 113: 104-9.

5. Murphy MP, Fishman P, Short SO, et al. Health care utilization and cost among adults with chronic rhinosinusitis enrolled in a health maintenance organization. Otolaryngol Head Neck Surg. 2002; 127(5):

6. Lund VJ, Black JH, Szabo LZ, et al. A Efficacy and tolerability of budesonide aqueous nasal spray in chronic rhinosinusitis patients. Rhinology 2004; 42(2): 57-62.

7. Snidvongs K, Kalish L, Sacks R, et al. Topical steroid for chronic rhinosinusitis without polyps. Cochrane Database Syst Rev. 2011: CD009274.

8. Kalish L, Snidvongs K, Sivasubramaniam $\mathrm{R}$, et al. Topical steroids for nasal polyps. Cochrane Database Syst Rev. 2012 Dec 12: 12:CD006549.

9. Snidvongs K, Chaowanapanja $P$, Aeumjaturapat $S$, et al. Does nasal irrigation enter paranasal sinuses in chronic rhinosinusitis? Am J Rhinol 2008; 22(5): 483-6.

10. Hopkins C, Browne JP, Slack R, et al. The national comparative audit of surgery for nasal polyposis and chronic rhinosinusitis. Clin Otolaryngol. 2006; 31(5): 390-8.

11. Ragab SM, Lund VJ, Scadding G. Evaluation of the medical and surgical treatment of chronic rhinosinusitis: a prospective, randomised, controlled trial. Laryngoscope 2004; 114(5): 923-30.

12. Harvey RJ, Goddard JC, Wise SK, et al. Effects of endoscopic sinus surgery and delivery device on cadaver sinus irrigation. Otolaryngol Head Neck Surg. 2008; 139(1): 137-42.

13. Grobler A, Weitzel EK, Buele A, et al. Pre- and postoperative sinus penetration of nasal irrigation. Laryngoscope 2008; 118(11): 207881.

14. Murr $A H$, Smith $T L$, Hwang PH, et al. Safety and efficacy of a novel bioabsorbable, steroid-eluting sinus stent. Int Forum Allergy Rhinol. 2011; 1(1): 23-32.

15. Forwith KD, Chandra RK, Yun PT, et al. ADVANCE: a multi-site trial of bioabsorbable steroid-eluting sinus implants. Laryngoscope 2011; 121(11): 2473-2480.
16. Marple BF, Smith TL, Han JK, et al. ADVANCE II: a prospective, randomized study assessing safety and efficacy of bioabsorbable steroid-releasing sinus implants. Otolaryngol Head Neck Surg. 2012; 146(6): 1004-1011.

17. Melroy CT, Kuhn FA. Safety of Ethmoid Sinus Drug-Eluting Catheter Insertion. Ann Otol Rhinol Laryngol. 2009; 118(10): 708-713.

18. Taulu R, Numminen J, Bizaki A, et al. Imageguided, navigation-assisted Relieva Stratus MicroFlow Spacer insertion into the ethmoid sinus. Eur Arch Otorhinolaryngol. 2015; 272(9): 2335-40.

19. Catalano PJ, Thong M, Weiss R, et al. The MicroFlow Spacer: A Drug-Eluting Stent for the Ethmoid Sinus. Indian J Otolaryngol Head Neck Surg. 2011; 63(3): 279-84.

20. Fokkens W, Lund V, Mullol J, et al. European position paper on rhinosinusitis and nasal polyps 2007. Rhinology Suppl. 2007; (20): $1-136$

21. Finnish current care guideline for sinusitis. Published 26 September 2013. Available from: http://www.kaypahoito. $\mathrm{fi} / \mathrm{web} / \mathrm{english/guidelineabstracts/}$ guideline? id=ccs00022 Accessed 17 January 2016

22. Minim: Allocation by minimisation in clinical trials. Available from: https://wwwusers.york.ac.uk/ mb55/guide/minim.htm Accessed 17 January 2016

23. Hopkins C, Gillet S, Slack R, et al. Psychometric validity of the 22-item Sinonasal Outcome Test. Clin Otolaryngol. 2009 Oct; 34(5): 447-54.

24. Numminen J, Dastidar $\mathrm{P}$, Heinonen $\mathrm{T}$, et al, Reliability of acoustic rhinometry. Respir Med. 2003; 97(4): 421-7.

25. Straszek SP, Schlüssen V, Sigsgaard T, et al. Reference values for acoustic rhinometry in decongested school children and adults: the most sensitive measurement for change in nasal patency. Rhinology 2007; 45(1): 36-9.

26. Lund VJ, Scadding GK. Objective assessment of endoscopic sinus surgery in the management of chronic rhinosinusitis: an update. J Laryngol Otol. 1994; 108(9): 74953.

27. Catalano PJ, Thong M, Garg V. The MicroFlow Spacer: a drug-eluting stent for the ethmoid sinus. Operat Tech Otolaryngol Head Neck Surg. 2009; 20: 108-113

28. Derendorf H, Meltzer EO. Molecular and clinical pharmacology of intranasal corticosteroids: clinical and therapeutic implications. Allergy 2008; 63(10): 1292-1300

29. Instructions for use of Relieva Stratus MicroFlow Spacer, MenloPark. Available from: http://acclarent.ru/sites/default/ files/speyser_i_buzh_relieva_stratus_dlya_ reshetchatogo_labirinta.pdfAccessed 17 January 2016

30. Sjogren PP, Parker NP, Boyer HC. Retained drug-eluting stents and recalcitrant chronic rhinosinusitis: A case report. Allergy Rhinol (Providence) 2013; 4(1): 45-8.

31. MAUDE Adverse Event Report: Acclarent, INC. Relieva Stratus deployment guide. U.S. Food and Drug Administration. Available from: http://www.accessdata.fda.gov/ scripts/cdrh/cfdocs/cfMAUDE/detail. cfm?mdrfoi__id=2647842 Accessed 17 January 2016

Rami Taulu

Department of Otorhinolaryngology

Tampere University Hospital

Teiskontie 35, Box 2000

33521 Tampere

Finland

Tel: +358331169456

E-mail: rami.taulu@pshp.fi 